\title{
La construcción del capital social en los egresados de la Facultad de Artes Visuales (FAV) en Monterrey, México
}

\author{
Social Capital Construction in Graduates \\ from Facultad de Artes Visuales (FAV) Located \\ in Monterrey, México
}

\author{
Tania Gissel Alonso González \\ César Morado Macías
}

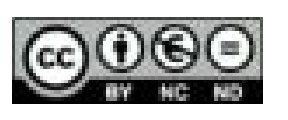

Esta obra está bajo una licencia Creative Commons Reconocimiento-No comercial-Sin Obra Derivada 


\title{
La construcción del capital social en los egresados de la Facultad de Artes Visuales (FAV) en Monterrey, México ${ }^{1}$

\author{
Social Capital Construction in Graduates \\ from Facultad de Artes Visuales (FAV) Located \\ in Monterrey, México
}

\author{
Tania Gissel Alonso González ${ }^{2}$ \\ Universidad Autónoma de Nuevo León \\ México \\ César Morado Macías ${ }^{3}$ \\ Universidad Autónoma de Nuevo León \\ México
}

Recibido: 12 de setiembre de 2018 Aprobado: 13 de febrero de 2019

\section{Resumen}

El presente artículo surge a partir de una investigación de tesis doctoral que busca describir y analizar los elementos que componen el capital social de los artistas visuales y sus etapas en el proceso de construcción. También interesa conocer cuál es el papel del capital social en su proyección profesional y cómo esto se vincula al desarrollo artístico y cultural de una ciudad industrial como Monterrey. A través de una metodología cualitativa se explora este capital en los artistas visuales egresados de la Facultad de Artes Visuales (FAV), al ser las entrevistas semiestructuradas la

1 Este artículo se desprende de la investigación titulada "Los artistas visuales en Monterrey. El capital social de los egresados de la Facultad de Artes Visuales (1985-2015)".

2 Candidata a doctor por la Facultad de Filosofía y Letras en el programa de Doctorado en Filosofía con acentuación en Estudios de la Cultura por la UANL. Becaria del Consejo Nacional de Ciencia y Tecnología (CONACYT). Correo electrónico: taniag.algzz@gmail.com

${ }^{3}$ Profesor e investigador de la Facultad de Filosofía y Letras de la UANL. Miembro del cuerpo académico consolidado Estudios Sociales e Históricos del noreste de México. Miembro del Sistema Nacional de Investigadores en México. Correo electrónico: cesarmorado614@gmail.com 
herramienta para desarrollar el proyecto, que se sostiene bajo un argumento central: la FAV como un pilar para la construcción del capital social de los artistas visuales de Monterrey. Desde este panorama, el principal objetivo del artículo es mostrar una síntesis de los resultados de investigación, lo cual posibilita la definición de los aspectos en el proceso de construcción de capital social, encontrados en los artistas visuales.

Palabras clave: capital social; artistas visuales; Pierre Bourdieu; Monterrey, México; Facultad de Artes Visuales

\begin{abstract}
This paper arises from a doctoral thesis research that seeks to describe and analyze the elements that make up the social capital of visual artists, as well as their stages in the construction process, what is the role in their professional projection and how this gets linked to the artistic and cultural development of an industrial city like Monterrey. Through a qualitative methodology the social capital of the visual artists graduated from FAV is explored, being the semi-structured interviews the tool to develop the project, which is held under the central argument: FAV is the pillar for the construction of social capital of the visual artists of Monterrey. From this perspective, the main objective of the article is to show a synthesis of the research results, which enables the definition of the aspects of the process of construction of social capital found in visual artists.
\end{abstract}

Key Words: social capital; visual artists; Pierre Bourdieu; Monterrey, México; School of Visual Arts 
La construcción del capital social en los egresados de

Artículos

la Facultad de Artes Visuales (FAV) en Monterrey, México

\section{Introducción}

La ciudad de Monterrey, capital del estado de Nuevo León, en la región noreste de México, se ha destacado por su liderazgo en el sector industrial, convirtiéndose en la segunda zona de mayor desarrollo económico del país. Situada en medio de un paisaje agreste, esta urbe se ha consolidado como una ciudad de negocios, no solo a nivel nacional, sino también internacional. En la conferencia Paisaje industrial de Monterrey: el discurso sobre la nueva imagen de la ciudad (2015) el historiador, César Salinas, expone que la imagen actual de Monterrey se definió en torno a las industrias que aparecieron en la ciudad y el discurso que se promovió entre sus trabajadores. Comenzó a ser descrita como una ciudad con cultura del trabajo. Monterrey se considera la capital industrial del país, por el desarrollo económico que vive, desde hace un siglo, vinculada a la economía de frontera con Estados Unidos de América (EUA), la más poderosa del mundo. Sin embargo, el auge económico no se tradujo en desarrollo artístico hasta hace muy poco.

La producción y el discurso artístico tuvieron un crecimiento paulatino, pero, fue en la segunda mitad del siglo pasado cuando prosperaron varios proyectos afines, entre ellos, la creación de museos, diversificación de talleres y el surgimiento de instituciones de educación superior. Un hecho que reafirma este interés en la ciudad es el de profesionalizar, por primera vez el campo audiovisual, en 1983, a través de la fundación de la Facultad de Artes Visuales (FAV de la Universidad Autónoma de Nuevo León (UANL). Al tomar en consideración a la FAV como referente en la ciudad, en cuanto a la formación de artistas visuales, se podrá describir la proyección y posicionamiento de sus egresados, a través de las relaciones logradas durante la formación académica. Puntualmente, este texto aborda como eje central los aspectos de construcción del capital social, el cual es un recurso relacional del agente, a partir de los elementos que sus relaciones poseen. Este concepto integra las dinámicas relacionales en la explicación de los fenómenos sociales, Pierre Bourdieu (1980) lo perfila como un medio para conocer la trayectoria de un agente social. Por esta razón, se emplea para la comprensión del proceso de construcción en el campo del arte, con el objetivo de indagar en la proyección profesional de los artistas visuales egresados de FAV.

El habitus (Bourdieu, 1972) produce y reproduce un conjunto de comportamientos y acciones, como noción articuladora de la dimensión histórica y de las trayectorias sociales dentro de los campos. El habitus es reproducido por una serie de mecanismos dentro del capital social, es decir, los intercambios que mantienen, refuerzan y transforman las redes de relaciones contingentes a relaciones reconocidas. En la FAV se estimula el habitus de los artistas visuales, lo cual determina las prácticas sociales, que forman, estructuran y participan en la construcción del capital social. De acuerdo a Bourdieu es el capital social lo que mantiene el habitus, a través de las disposiciones que el grupo les inculca y les refuerza en sus redes relaciones.

ESCENA. Revista de las artes, 2019, Vol. 79, Núm. 1 (julio-diciembre), pp. 119-144 
Analizar el capital social de los artistas visuales, a partir de la institución de mayor tradición que les ha formado académicamente, conlleva descubrir el proceso de creación de las relaciones y de los fundamentos que sostienen a este grupo. Además de cómo esto se vincula al desarrollo artístico y cultural de una ciudad industrial como Monterrey. El carácter del trabajo sugiere restringir la búsqueda a un determinado periodo y a un lugar específico. Por tal motivo, se tomará en cuenta a los egresados de la licenciatura en artes visuales de la FAV, desde 1985 hasta 2015. Se seleccionará un grupo de egresados para realizar entrevistas acerca de su práctica artística y trayectoria, para enfocar el análisis en la formación del capital social y, así, poder dar seguimiento al recorrido y las conexiones de los artistas en la ciudad.

El presente artículo tiene como objeto dimensionar el capital social de los artistas visuales. Por lo tanto, visualizar su construcción a partir de la escuela de artes y cómo estimula o favorece su proyección profesional. Este texto aporta datos y parámetros suficientes para lograr un primer acercamiento al sector artístico local, en términos de las redes de relaciones que explican su inserción laboral. Además, aporta una serie de referencias que combaten falsas percepciones y conclusiones comunes del arte local.

\section{El capital social desde la perspectiva de Pierre Bourdieu}

Pierre-Félix Bourdieu trató de forjar la teoría del capital social como una base clave para comprender la naturaleza de la estructura social, en cuanto a su lógica de reproducción de clases sociales y configuración de poder político. No obstante, otros de sus aparatos teóricos fueron los que iluminaron estos fenómenos con una mayor claridad. Por su parte, el capital social se ha configurado como un instrumento teórico y analítico, a través del cual se explican procesos dinámicos que generan grupos diferenciados. Es decir, aunque en este proyecto de investigación la estratificación social de la comunidad artística no es el eje central, sí lo son los distintos espacios que ocupan los artistas visuales. Así, a partir de la misma plataforma (FAV), se ostentan distintos lugares de influencia y proyección. Desde este enunciado se encuentra circunscrito el proyecto a la línea de Pierre Bourdieu.

En 1980 Pierre Boudieu definió el capital social como el conjunto de los recursos reales o potenciales que se vinculan con la posesión de una red duradera de relaciones más o menos institucionalizadas de interconocimiento y de interreconocimiento. En otros términos, con la pertenencia a un grupo, como conjunto de agentes que no están solamente dotados de propiedades comunes (susceptibles de ser percibidas por el observador, por los otros o por ellos mismos), sino que están también unidos por lazos permanentes y útiles ( $p$. 221). Este esquema de relaciones nace y se sustenta en los intercambios recíprocos, materiales y simbólicos, lo cual provee de recursos para lograr fines personales. No obstante, 
La construcción del capital social en los egresados de

Artículos

la Facultad de Artes Visuales (FAV) en Monterrey, México

el aprovechamiento de los beneficios que otorga la pertenencia a un grupo o la movilización de los recursos ofrecidos, para concretar un interés particular, tiene que ver con el criterio y la posición de cada actor.

Bourdieu asume el capital social como una herramienta para analizar el proceso bajo el cual este tipo de capital se genera, se comparte o se reproduce. Dicha postura enfatiza en la necesidad de comprender la dinámica del capital social, donde el sujeto es el eje medular que puede instaurar, conservar o destruir este tipo de capital. Como resultado se generan vínculos, que se extraen del grupo en el que se está inserto, y los moviliza con base en los alcances de las relaciones implicadas en esa red.

Jorge Ramírez Plascencia en el texto Tres visiones sobre capital social: Bourdieu, Coleman y Putnam (2005) subraya cuatro elementos clave que permiten la comprensión del capital social en Pierre Bourdieu: la pertenencia a un grupo, la existencia de relaciones de intercambio material y simbólico que se dan en su interior, su grado de institucionalización y los recursos que posee dicho grupo. Las relaciones que los artistas vayan formando durante su recorrido académico, con el objetivo de proyectar su carrera a un nivel profesional, pasan por el filtro de los intercambios materiales y simbólicos que Bourdieu plantea. Estos reúnen intereses, no solo entre personas, sino entre instituciones. Es decir, el hecho que un artista se conecte con un galerista, un curador o un colectivo de arte, lo hace compartir una serie de valores o recursos particulares a esa estructura del arte con la que pretende ligarse.

Disponer de una red relacional es una condición necesaria para el desarrollo de la carrera de un artista, pero dista de ser suficiente. El capital social no es un baza determinista, también hay que saber enriquecerlo, movilizarlo. Si la capacidad del artista para atrapar, incluso, provocar, las oportunidades constituye uno de los aspectos cruciales de las trayectorias comprobadas; el papel jugado por la variable institucional aparece tanto como esencial (Moureau \& Zenou, 2014, pp. 121-122). Asimismo, las estrategias para construir el capital social tienen como objetivo la perpetuación o el aumento de este. Zenou (2009) propone, en primer lugar, la instauración de relaciones sociales, a partir de la pertenencia a un grupo y el sostenimiento de estas orientadas hacia su utilización a corto o largo plazo. Es decir, la transformación de estos recursos en inversión social. Por ejemplo, a través de compromisos subjetivamente percibidos o institucionalmente garantizados, que se manifiestan en intercambios materiales o simbólicos. Por lo tanto, el sostenimiento de estas relaciones se dirige a mantener y aumentar el reconocimiento social. Por su parte, Bourdieu señala al capital social como las relaciones movilizables por el agente y los recursos que él controla.

Dentro de este contexto se retrata la posibilidad de analizar esta herramienta teórica, para mejorar su instrumentalización en una geografía industrial y de condiciones mayormente áridas. La forma en la que opera el capital social y sus beneficios en las trayectorias de los 
artistas y su influencia en el desarrollo de Monterrey, no solo en la cultura, sino, también, al fortalecer otra dimensión de su economía, son aspectos que se desglosan de este modelo teórico a la luz de las entrevistas realizadas.

\section{El método}

A la luz del problema a investigar en este proyecto se optó por la metodología cualitativa. Por medio de un diseño de investigación fundamentado en la teoría, el cual trata de explicar, mediante la metodología inductiva, la interpretación de significados desde la realidad social de los individuos. Esto con el propósito último de crear una teoría que explique el fenómeno del estudio (Vivar, Arantzamendi, López-Dicastillo \& Gordo, 2010). Dentro de este esquema, las entrevistas semiestructuradas fueron el canal para acercarse a los artistas visuales, los actores sustantivos en este particular contexto, para así obtener la descripción del capital social. La información obtenida fue registrada mediante una grabación, ya que esta herramienta facilita la transcripción posterior, a la cual todos los entrevistados otorgaron consentimiento. Asimismo, los comentarios y observaciones se adjuntaron por escrito en un cuaderno destinado a esta tarea. Este material se anexó junto a las transcripciones de las entrevistas y conversaciones informales previas o posteriores a estas, que fueron relevantes para la investigación.

Después, se establecieron ejes de análisis (pertenencia al grupo, recursos, vínculos e intercambios) de acuerdo al modelo teórico del capital social (Bourdieu, 1980; Ramírez, 2005; Zenou, 2009), los cuales se vinculan con una o varias preguntas del cuestionario utilizado en las entrevistas. En cuanto a este recurso, los cuestionamientos fueron una guía, ya que cada diálogo tomó su propio rumbo y, en algunas ocasiones, no era necesario abordar las temáticas a partir de una pregunta, debido a que el entrevistado sumaba estos puntos en sus respuestas. En algunos otros casos, las respuestas eran concisas y fue necesario echar mano de todos los recursos previstos. Este registro permitió explorar el recorrido y las conexiones de los artistas con agentes dentro y fuera del circuito local. Así como la proyección conseguida a través de las redes de contacto formadas durante su paso por la FAV.

La selección de los entrevistados se realizó por medio de muestreo en cadena. La razón por la que no se puede realizar un muestreo aleatorio es simple, debido a que la legislación mexicana vigente declara que los expedientes académicos constituyen información confidencial, por lo tanto, no son públicos. De tal manera, se procedió a buscar los primeros contactos a través de la Subdirección de Posgrado de FAV, ya que realicé mis estudios de maestría en esta dependencia y algunos de mis maestros fueron egresados de la licenciatura con la que estoy trabajando para el proyecto. Ellos fueron quienes me vincularon hacia otros sujetos relevantes para el tema de investigación.

ESCENA. Revista de las artes, 2019, Vol. 79, Núm. 1 (julio-diciembre), pp. 119-144 
Los artistas visuales contactados fueron en total doce. Todos con distintas prácticas artísticas, edad, generación de egreso y proyectos profesionales, como se puede visualizar en el siguiente diagrama. Se buscó intencionalmente esta panorámica para obtener perspectivas desde distintos horizontes del arte, con la finalidad de explorar diferentes caminos (si es

Diagrama 1. Artistas entrevistados

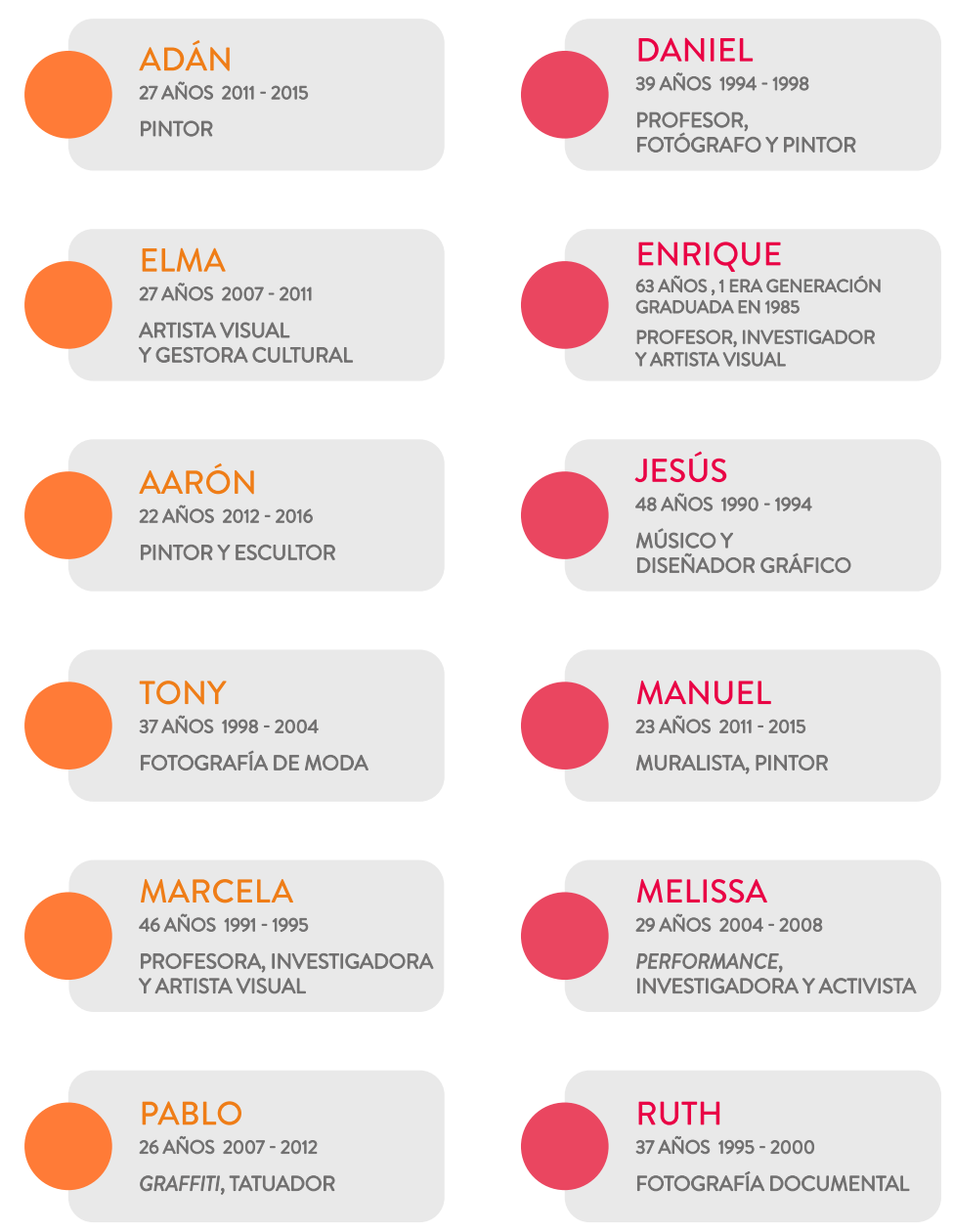

Fuente: Elaboración propia.

que hubiera) en la construcción del capital social. Es importante mencionar que, por cuestión de confidencialidad solo se muestra el primer nombre de los artistas.

Las entrevistas se realizaron durante los años 2016 y 2017. Estas facilitan la conexión entre la experiencia y subjetividad de los artistas con su mirada crítica y la evaluación de su historia en la FAV, así como la vinculación con su trayectoria profesional. Mientras que los hallazgos cualitativos fueron sistematizados en ejes de análisis de acuerdo al modelo del capital social. La unidad de información fueron los egresados de la licenciatura en Artes Visuales de la Facultad de Artes Visuales de la Universidad Autónoma de Nuevo León en el periodo 19852015. Mientras que la unidad de análisis considerada fueron sus opiniones y perspectivas en la construcción del capital social. Dentro de este apartado es preciso señalar que, la voz de los artistas está fundamentada en su experiencia como estudiantes de FAV y en cómo esto se ha traducido en su recorrido pro-
fesional. Por tal motivo, recurren a sus anécdotas como estudiantes. No obstante, todos, en el momento de la entrevista, habían concluido sus estudios y están en la categoría de egresados de esta licenciatura.

ESCENA. Revista de las artes, 2019, Vol. 79, Núm. 1 (julio-diciembre), pp. 119-144 
Un aspecto clave para la construcción del guión de entrevista fueron las referencias que se buscaron en tesis doctorales de temas afines al capital social, así como el estudio que llevaron a cabo Néstor García Canclini y Ernesto Piedras Feria (2013) titulado Jóvenes creativos, estrategias y redes culturales. Estos eslabones fueron centrales para el desarrollo del instrumento metodológico, pues considero que la madurez de la práctica científica social se ha mantenido en un entramado de estudios y posibilidades que se conectan y nutren sus espacios compartidos, siendo estos sus pilares filosóficos, teóricos y metodológicos. De tal manera, esta investigación no puede nacer en el aislamiento, sino en plena comunicación con sus antecedentes históricos y, sobre todo, en constante atención a sus necesidades específicas.

Para dictaminar los ejes de análisis basados en el capital social de Pierre Bourdieu se usó como guía la propuesta de estudio de este concepto que hizo Benoit Zenou (2009) en Le Capital Social comme un Potentiel d'Interaction Coopérative: le cas des Relations Intergénérationnelles Familiales. Su propuesta nace del comprender las motivaciones y los efectos del capital social, para determinar sus componentes. Zenou parte de la comprensión de Bourdieu, donde retoma los principios marxistas y supone una estructuración social entendida en los modos de producción y reproducción de capital. Con este enfoque individual que Bourdieu le concede y que Zenou aplica, el análisis se dirige hacia los indicadores que explican las características de los recursos y su estructura relacional. De tal forma, el capital social explica las relaciones sociales y, por lo tanto, el lugar que ocupa cada agente.

A continuación se abordan los cuatro ejes de análisis del capital social, los cuales se observaron como los aspectos de su construcción. Cada una de ellos comprendió distintas aplicaciones, dependiendo del contexto de cada artista. Además, cada aspecto agrupa una serie de tópicos que completan la ilustración de cómo y a qué se debe el capital social. A manera de síntesis y para facilitar la lectura, se presenta el siguiente diagrama con las conclusiones de cada uno de estos aspectos, a partir de las ideas y experiencias que los artistas compartieron. 
Diagrama 2. Construcción del capital social en artistas de artes visuales.
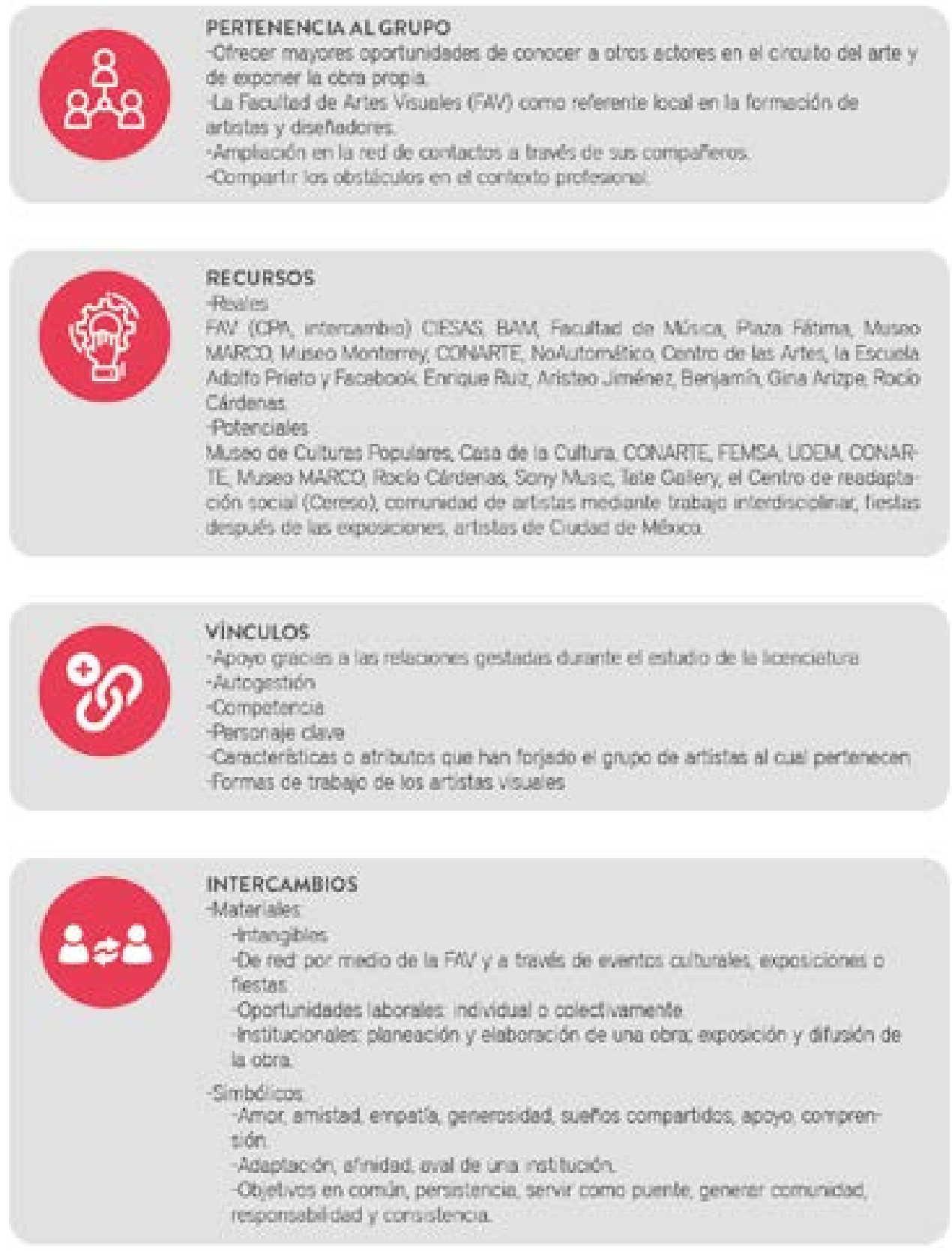

Fuente: Elaboración propia.

ESCENA. Revista de las artes, 2019, Vol. 79, Núm. 1 (julio-diciembre), pp. 119-144 


\section{Aspecto 1. Pertenencia al grupo}

El primer rubro se refiere a la pertenencia que ellos consideran tener con el grupo de artistas visuales. En las entrevistas, la primer pregunta es la que se dirigía a este aspecto. Nueve de los doce entrevistados concluyeron que estudiar en FAV sí favorece el sentido de pertenencia a la comunidad de artistas. Además, ofrece mayores oportunidades de conocer a otros actores en el circuito del arte y de exponer la obra propia. Adán comenta "si te da algo de pertenencia porque estás siempre relacionando con los de la universidad; con lo de FAV logras hacer amigos, contactos" (comunicación personal, 22 de febrero de 2017). Marcela y Ruth coinciden en el peso que tiene la FAV como referente local en la formación de artistas y diseñadores. Esto implica un atributo importante en los egresados de la primera escuela que instituyó profesionalmente las Artes Visuales en Monterrey.

Enrique y Pablo comentan que ellos observan este proceso de identificación con los demás artistas en el acercamiento que la FAV ofrece de forma implícita al mundo del arte, como un aspecto clave del proceso. Sin embargo, una identificación consolidada tiene que ver más con cada uno de los individuos y cómo resuelven su trayectoria entre las complejas fuerzas del circuito. Así como la forma de conformar un discurso, práctica propia y obtener lazos a mediano y largo plazo. Esta situación se relaciona con el caso de Jesús, quien se ha desarrollado en el Arte, la Publicidad, el Diseño editorial e industrial y la Música. En este último rubro fue al que se dedicó en los años siguientes a su egreso, ya que junto con otros compañeros de la FAV conformaron el grupo Cabrito Vudú y formaron parte del movimiento conocido como "Avanzada Regia". Ignacio Corona en La Avanzada Regia: Monterrey's Alternative Music Scene and the Aesthetics of Transnationalism (2011) la define como la corriente estética que se ubica entre la tradición y la modernidad, la cual muestra cómo la producción cultural puede cambiar la dicotomía existente entre el centro y la periferia, que ha favorecido a la Ciudad de México en el imaginario nacional. A finales de los noventa surgió este movimiento musical, el cual involucró grupos como Control Machete, Plastilina Mosh, Kinky, Cabrito Vudú, Jumbo, El Gran Silencio, Inspector, entre otras. Todos ellos combinan complejos procesos de producción trasnacional con un profundo sentido de identificación territorial, centrado en la noción cultural de ser regiomontano (gentilicio de Monterrey, México).

Otra de las ventajas del sentido de identificación la menciona Daniel. Él habla de una ampliación en la red de contactos a través de sus compañeros (con los que estudió en el aula o con los que compartió la generación, ya que en FAV existen otras licenciaturas) no solo de forma local, sino regional y nacionalmente. Por lo tanto, este se legitima y se extiende a través de las distintas implicaciones sociales, geográficas y contextuales del mundo del arte (Dickie, 1997). Daniel comenta "si se genera una identificación cuando eres egresado de FAV porque conoces gente que está inserta en el medio, o que se está insertando, y los 
contactos se amplían cuando cada compañero regresa a su ciudad, los que son foráneos, o con los que son de aquí, pero se van a otras partes del país a trabajar" (comunicación personal, 30 de septiembre de 2016). En El capital social, el arte contemporáneo y las carreras (2014), Nathalie Moureau y Benoit Zenou afirman que:

el papel de la escuela más allá de ofrecer el panorama de técnicas artísticas y discursos teóricos, se convierte en un eslabón para los artistas con una red de relaciones tejidas entre actores en el mundo del arte, de igual manera se forja el universo profesional de estos dotándolos de códigos, normas y valores que son característicos de este arte "institucional" y le permiten dirigirse más fácilmente a las personas que forman parte de eso (p.117).

Finalmente, Tony fue el único en ofrecer un rotundo no como respuesta a este cuestionamiento. Argumenta las dificultades del circuito local como obstáculo para que el gremio se fortalezca al incluir a nuevos artistas, proyectos nuevos o gente joven. "No, en lo absoluto, porque en FAV estando en Monterrey se maneja un circuito del arte muy cerrado. Tienes que buscar más espacios y trabajar por tu parte. Cuando sales de Monterrey, y ves desde afuera el circuito del arte de Monterrey, este no importa en absoluto" (Tony, comunicación personal, 29 de septiembre de 2016).

El capital social exige del artista visual estar en un grupo. Este no puede ser analizado en el aislamiento de un solo actor, sino en la red de relaciones y en el aprovechamiento de los beneficios que esta pueda conllevar para cada sujeto. En los ejemplos se ilustra un primer aspecto del capital social, el cual se refiere a la pertenencia de grupo y a la capacidad de reconocimiento con él. Pero, ¿cómo es determinante la FAV en este proceso? Los egresados entrevistados la definen no solo en atributos positivos que cohesionan a quienes recorrieron juntos las aulas y quienes han significado algunos de sus primeros contactos en la escena profesional. A través de ella, también, se comparte y se visualiza un contexto profesional adverso (escasas garantías sociales, trabajos temporales y nulas políticas institucionales) y una serie de obstáculos locales alrededor de la práctica artística.

\section{Aspecto 2. Recursos}

Este segundo rubro de análisis se divide en reales y potenciales. Todos los entrevistados enlistaron a la FAV, sus profesores y respectivos compañeros de generación en esta sección, primordialmente, en los recursos reales. No obstante, el circuito del arte en el que cada uno se desarrolla incide en las diferencias existentes entre los recursos de los entrevistados. 
Los recursos reales se refieren a las relaciones interpersonales; son los recursos que desean transferir o los que ponen al alcance de los demás (Zenou, 2009). Entre los más comentados se encuentran ciertos espacios con los que FAV ha tenido convenios o relaciones estrechas para ofrecer un lugar para exposiciones. Entre ellos, el Centro de las Artes, el Museo de Arte Contemporáneo de Monterrey (MARCO), el Centro de Investigaciones y Estudios Superiores en Antropología Social (CIESAS), el Centro Cultural BAM, la Facultad de Música, Plaza Fátima, el Museo Monterrey, Consejo para la Cultura y las Artes de Nuevo León (CONARTE), NoAutomático y la Escuela Adolfo Prieto.

Otro común denominador entre los entrevistados fue la importancia de Facebook y redes sociales personales como un recurso. La relevancia de la FAV también se muestra al encontrar una serie de nombres que varios de los entrevistados mencionaron como parte de sus recursos, algunos fueron sus maestros o compañeros. Entre ellos, Enrique Ruiz, Aristeo Jiménez, Benjamín Sierra, Celeste Flores, Aarón de León, Martín Leal, Tony Solís, Manuel Emilio Salazar, Gina Arizpe, Ruth Rodríguez, Rocío Cárdenas.

En esta misma línea se encuentra el caso de algunos que han hecho contactos muy específicos en sus trayectorias, por ejemplo, Adán y Aarón en el Centro de Producción Audiovisual (CPA). Durante sus años como estudiantes, en este espacio de la facultad se empezaron a reunir con la excusa de limpiarlo y utilizar las herramientas que ahí se encontraban. Muchos de ellos no tenían en sus casas el espacio para trabajar en sus obras, por esta razón, se dedicaron a rescatar esta área de la escuela. "Se empezó a generar comunidad a través del CPA, varios platicamos sobre el porqué no ibamos a limpiar el tórculo. Empezamos a ir a un espacio para hacer grabado, escultura monumental. Fuimos a limpiar el CPA porque estaba lleno de bancos. Nos empezamos a reunir, a platicar y a hacer grabado en el CPA y notábamos que era algo muy bueno. Convivíamos, comíamos juntos, dibujábamos" (Adán, comunicación personal, 2017).

Posteriormente, se volvió un punto de encuentro entre las generaciones recientes (2012-2016) donde se impartían clases alumno-alumno con estudiantes de la misma FAV o de la Facultad de Música (vecina de la FAV en el campus Mederos de la UANL). Se dieron a la tarea de aprovechar ese espacio para convivir, realizar proyectos personales, aprender nuevas técnicas de trabajo y eso se convirtió en amistades a largo plazo. Así como el inicio de objetivos profesionales que cada uno se trazó y, actualmente, están materializándose. Adán también comentó una oportunidad que solo pudo conseguir a través de la FAV: un intercambio académico en la Universidad Complutense de Madrid, lo cual enlistó como un recurso real. Allá tuvo la oportunidad de generar nuevas redes de contacto y conocer otra forma de trabajar e insertarse en otros circuitos del arte. Adán también comenta: 
La construcción del capital social en los egresados de

Artículos

la Facultad de Artes Visuales (FAV) en Monterrey, México

Ahora tengo de roomie a otro chavo de la FAV y a una fotógrafa. Vivo con ellos porque tenemos un proyecto que era lo que hacíamos en el CPA, ahora que ya salimos de la FAV, necesitamos un espacio. Yo me dedico a dar talleres, a hacer mi obra, a hacer encargos, pero este espacio lo queremos adaptar para hacer exposiciones. Lo de los contactos sirve porque hay maestros que nos dan retroalimentación, nos dicen qué mejorar y por dónde movernos. Por otro lado, el intercambio me ayudó muchísimo, si no hubiera sido por FAV no hubiera conocido Madrid, no hubiera montado todo esto. Sí sirve esto de FAV, porque hay gente que compra obra, o los galeristas te conocen o gente que te conoce pero por tu trabajo, porque en FAV se da como una pequeña prueba de lo que será la vida real (comunicación personal, 2017).

Adán y Aarón comparten la opinión positiva acerca de los viajes de estudio a la capital del país, donde se dedicaban a visitar museos, talleres o exposiciones. Adán (comunicación personal, 2017) apunta "también en los viajes de estudio que se armaban a la Ciudad de México, siempre iba el coordinador y otro profesor, ahí se construían mejores lazos con ellos y, a veces, te presentaban a otros artistas. En algunas ocasiones, también te daban sus contactos para que nos dejaran exponer en galerías o espacios culturales". Moureau \& Zenou (2014) sostienen que

Entre los que frecuentaron las escuelas de Bellas Artes, los artistas que tienen las carreras "más 'fecundas", en el curso de sus estudios, han tejido lazos con algunos actores claves. Así, el hecho de ser asistente de un artista reconocido, durante, o a la salida de la escuela, le permite al alumno gozar de las redes de esta persona mayor y ser presentado a actores múltiples del mundo del arte (críticos, galeristas, etc.). Le ofrece además la oportunidad de ver se práctica en el 'oficio social' del artista ... Para los artistas que no han gozado de estas oportunidades, los profesores de la escuela son quienes ofrecen los espacios potenciales ... La rapidez con la que un joven talento sepa aprovechar esas oportunidades para enriquecer su red y salir del territorio se convierte en un elemento que determinará su carrera (p. 117).

Finalmente, Elma menciona en sus recursos reales a artistas alternativos, Facebook, galerías, instituciones educativas, la galerista Emma Molina, Martín Leal (exprofesor) y otra artista visual Gina Arizpe.

Como gestora hice buenos contactos a través de maestros, pero tú tienes que hacer presencia, en eventos, fiestas, proyectos, etc. Como gestora no tengo tanto problema, como artista si he tenido más conflicto, ya que mi línea de producción no tiene mucha visión de venta o galería, es algo más particular, por eso no estoy tocando muchas puertas. Hago poesía visual y literatura electrónica. También he hecho instalación, arte objeto y fotografía. Me he dedicado a conectar a los artistas emergentes con más gente, soy como un puente. No he buscado mucho exponer mi propia obra (Elma, comunicación personal, 26 de setiembre de 2016).

ESCENA. Revista de las artes, 2019, Vol. 79, Núm. 1 (julio-diciembre), pp. 119-144 
Así, la comunidad de artistas visuales funciona como un campo (desde una perspectiva bourdieuana), con sus relaciones dinámicas, inversión social y un volumen de recursos poseído por diferentes agentes de manera asimétrica. Ser egresado de una escuela de artes, en este caso FAV, es un instrumento de apropiación del capital social, de los mecanismos que hacen funcionar el campo los beneficios que procura. A través de estas experiencias narradas se sustenta esta afirmación.

La última parte de este rubro se refiere a los recursos potenciales (Zenou, 2009), los cuales son recursos no relacionales. Es decir, compromisos, deudas; recursos que corresponden a todos los que posee un agente gracias a sus relaciones. En este apartado, las respuestas, aunque se repitieron, fueron variadas, otras tienen que ver con el nicho específico en que el artista se ha desarrollado. Entre ellas se encuentran el Museo de Culturas Populares, la Casa de la Cultura, CONARTE, la Universidad de Monterrey (UDEM), el Museo MARCO, Rocío Cárdenas, Sony Music, Tate Gallery, el Centro de Reinserción Social (Cereso), la comunidad de artistas mediante trabajo interdisciplinar, las fiestas después de las exposiciones y los artistas de Ciudad de México.

La posibilidad de disponer de los recursos de un grupo se presenta como el segundo aspecto de construcción del capital social. Los artistas visuales comparten condiciones de vida que producen identificación social y apoyo en las actividades profesionales. Este apartado se refiere a la disposición de recursos gestada en los espacios compartidos (FAV). Dentro de las relaciones que conllevan los recursos reales y potenciales. Por un lado, se encuentra la deuda que ofrece obligación, reconocimiento, prestigio o fidelidad personal. Por otro, la red de respaldos y protecciones de la cual se puede recibir ayuda, un préstamo, o un lugar para exponer. Partiendo de este contexto, la discusión continúa hacia cuál es la movilización de estos recursos y cómo estimulan la conexión entre los egresados de FAV y z

\section{Aspecto 3. Vínculos}

Las implicaciones de los recursos se fortalecen en la ayuda mutua, lo cual se dirige hacia la creación de vínculos. Estos se definen como la inversión social que depende del agente. Conversaciones y acciones entre personas o grupos de personas orientadas hacia el intercambio de apoyos sociales, lo cual se moviliza como vínculos (Zenou, 2009). El primer eje sobre el que se reflexionó fue cómo consideraban que les había apoyado en su desempeño artístico, las relaciones gestadas durante el estudio de la licenciatura. Es decir, los contactos en el terreno del arte que a través de la facultad consiguieron. Manuel menciona la importancia de los contactos de alumnos con profesores que estén involucrados de forma activa en los circuitos del arte, que obtuvieron alguna beca, que hayan expuesto, que tengan su portafolio completo, que participen en algún colectivo, que conozcan de museografía o curaduría. En fin, que su conocimiento no se reduzca a lo meramente académico. 
La construcción del capital social en los egresados de

Artículos

la Facultad de Artes Visuales (FAV) en Monterrey, México

Son fundamentales. A nivel inmediato los compañeros que conocí en FAV, que no necesariamente producen, pero están en dependencias culturales. En segundo nivel los profesores con quienes tienes confianza y te ayudan a desarrollarte y algunos maestros, muy pocos, los que están vinculados con instituciones que te puedan profesionalizar (Manuel, comunicación personal, 07 de septiembre de 2016).

El siguiente eje de análisis se dirige hacia la autogestión y el emprendimiento de los artistas visuales. Es decir, las implicaciones de esta herramienta para crearse oportunidades laborales e insertarse en el mundo profesional del arte. Elma afirma que la autogestión es la primera estrategia y la más importante. Ella, como muchos artistas, ha balanceado su trabajo entre la producción y la gestión cultural. Elkin Rubiano (2013) examina el desarrollo de la autogestión en las generaciones de artistas recientes, al contrastarlo con el concepto de autonomía. Una nueva generación de artistas piensa: "gestiona o morirás". Generaciones atrás no se hubiera imaginado tal cosa, pues, en lugar de gestión se creía en el descubrimiento. Es decir, en el ser descubierto como artista. A grandes rasgos podríamos señalar un punto de partida y uno de llegada: el genio creador y el proveedor de contenido creativo; y algunos tránsitos: el artista como creador, el artista como productor, el artista como gestor. En otras palabras, el tránsito que va del genio individual (la impronta del maestro) a la comisión colectiva (los proyectos y el trabajo colaborativo).Pablo Núñez, quien se dedica al graffiti y al tatuaje, principalmente, coincide en la importancia de la autogestión al indicar que

La autogestión es fundamental. El hecho que fue por mis propios medios y mis propias ganas que empecé a pintar afuera, de esta cuestión de lo ilegal, que realmente no fue desde el principio que se me invitara a pintar o que se me diera permiso. Sí me han agarrado, pero es parte de la naturaleza de la práctica, y pues sí ha sido fundamental la autogestión, sobre todo porque buscaba revindicar cierto discurso al estar trabajando afuera en la calle, sin permiso (Pablo, comunicación personal, 28 de febrero de 2017).

El primer paso para crear y conservar un vínculo es el trabajo propio de forma consistente y con un argumento sólido, el cual se debe mostrar en la manufactura de la obra y en los temas propuestos. En este camino la autogestión es clave, ya que busca crear un discurso artístico propio. La práctica artística de cada uno está anclada a un discurso específico y se vuelve relevante para la agenda de uno o más circuitos artísticos. Cuando esta práctica se vuelve consistente y creadora de proyectos, se generan colaboraciones y las oportunidades se multiplican. Pablo opina

Tengo un buen amigo que trabaja en el Museo de Culturas Populares, pero él trabaja en la parte administrativa, y pues tener ese contacto si ha permitido que sucedan ciertas cosas. Se han abierto puertas. ¡Claro! Tampoco es compadrazgo puro. Mi trabajo es el que habla (Pablo, comunicación personal, 28 de febrero de 2017). 
Por su parte, Manuel argumenta: "la gente te abre la puerta para insertarte en un circuito, pero el que responde eres tú. Dependes de ti, de cómo muevas los contactos y las relaciones. Estás ahí porque saben tu trabajo, del compromiso que tienes" (comunicación personal, 2016). Mediante la enunciación de un proyecto profesional es posible situarse con una postura definida en la escena del arte. De tal forma, se presenta el trabajo colaborativo y la competencia, lo cual representa, aunque con diferentes matices, una fuente viva de relaciones. Tony señala:

Tiene que ser un equipo sólido y colectivo para que funcione, si quieres que tu ciudad o tu país sean un referente de la moda, del arte, tienes que hacer comunidad. Si quitas trabajo, no haces una industria, tienes que pensar en grande. Hay que hacer equipo, hacer comunidad, a los enemigos hacerlos aliados (comunicación personal, 2016).

Alfredo Herrera Pescador (2004) indica que el modelo social de Monterrey es muy competitivo y el campo artístico no se escapa de esto. Elma apunta a la competencia como una estrategia de diferenciación.

De afuera hacia adentro de Monterrey no nos podemos ver mucho si cada quien intenta encontrar el hilo negro. Necesitamos buscar una identidad homogénea, para poder darnos a conocer en otro lado, necesita haber más de lo mismo, y aquí cada quien hace cosas distintas, y si no nos unimos, nunca nos vamos a identificar. Todos aquí son muy fijados en tener una identidad propia, que no se parezca a la del compañero tal. Cada quien intenta jalar para su lado (Elma, comunicación personal, 2016).

Desde esta perspectiva, la competencia es un factor unificador que permite consolidar una personalidad artística local, con distintos matices. Sin embargo, mientras los artistas operan de forma individual y no reflexionan cómo sumar a la comunidad artística, el resultado es una débil agenda en la ciudad y fragmentación en los públicos. La galerista Emma Molina confirma este señalamiento. En entrevista con Benjamín Sierra (2004) comenta:

Aprendí en un lapso de 5 años y lo primero que me di cuenta fue que, uno de los grandes problemas del arte joven mexicano es que nuestra naturaleza y esencia como mexicanos es terriblemente individualista, un proyecto de arte necesita un consenso para lograr penetración ¿cómo es posible que Gabriel Orozco sea un proyecto tan bien logrado? Quizá porque Gabriel lo pudo leer muy a tiempo: en México todos se sacan los ojos. No se puede hacer un proyecto en Monterrey sin que el vecino venga y te aviente una pedrada. Supongamos que tengo a 4 o 5 artistas interesantes, propositivos, de gran futuro, como para que tengan un lenguaje universal, 
La construcción del capital social en los egresados de

Artículos

la Facultad de Artes Visuales (FAV) en Monterrey, México

lo que menos quiero es que los demás los vean, porque sé que van a venir y me los van a robar y me los van a sabotear y van a convencerlos de que ellos con fulano o sutano van a estar mejor que conmigo y me lo han hecho, lo mismo sucede en los museos, un ejemplo: en Londres un museo muestra la obra de Damien Hirst y 3 o 4 galerías también lo muestran simultáneamente como apoyo en donde todos ganan (Molina citada en Sierra, 2004, p. 49).

Otra de las líneas de análisis, dentro de los vínculos, fue algún personaje clave en el posicionamiento artístico, al servir como puente y conectar a los artistas con agentes afines a su entorno. En este sentido, Elma señala la importancia de las fiestas y eventos sociales como espacio clave en el desarrollo del posicionamiento profesional, así como para lograr fortalecer la red de contactos. Sin embargo, todos coinciden en la importancia de los profesores, no solo para la formación académica de los alumnos, sino como un referente directo de la forma de insertarse profesionalmente en los circuitos artísticos.

Nathalie Moureau y Benoit Zenou (2014) afirman que, en cuanto a los artistas plásticos, varios estudios han mostrado la importancia de las escuelas de Bellas Artes en el contacto de los alumnos con las redes profesionales. Entre ellos, La ocupación del artista (2004) de Françoise Liot, donde explica que las exposiciones realizadas, la presencia de los jurados en finales de año y el contacto con los responsables de instituciones constituyen elementos susceptibles de llamar la atención de un experto del mundo del arte sobre el trabajo del joven artista. De este modo, permite iniciar el reconocimiento del artista. Nathalie Heinich (1998) en El triple juego del arte contemporáneo concluye que, el papel de las redes de legitimación ha sido recalcado por el arte contemporáneo, en el cual el mundo museal no es el único espacio de circulación artística. En este sentido, Howard Becker (2009) en Los mundos del arte menciona que los convenios y las instituciones para las carreras de artistas en el siglo XIX son elementos centrales en el posicionamiento dentro del arte contemporáneo.

Los artistas visuales están condicionados por su localización en el espacio social y extraen de ella un valor determinado. No obstante, el contenido cooperativo y recíproco de las relaciones en el campo del arte es alto, debido a los equipos de trabajo y el intercambio mutuo entre instituciones e individuos. En este sentido, el penúltimo rubro que incide en la formación de vínculos se refiere a las características o atributos que han forjado el grupo de artistas, al cual pertenecen. Enrique cita el valor de la FAV como institución del arte que sostiene un imaginario particular y estimula proyectos e ideas (Dickie, 1997). No obstante, le asigna el papel protagónico al artista visual. Daniel asume que el común denominador del grupo de artistas es el reconocerse como miembro y apoyarse.

Entre compañeros nos apoyamos, para los artistas el trabajo interdisciplinar y la comunidad es muy importante. Aquí en FAV y con los de la Facultad de música 
son con los que se ha compartido la escuela, la licenciatura, proyectos, entre otros. La colaboración y el trabajo son claves (Daniel, comunicación personal, 2017).

Por su parte, Manuel comenta que dos de las características principales que aglutinan a los artistas es la persistencia y la capacidad de adaptarse. Uno de los detonadores de la vida asociativa es la asistencia a las exposiciones y presentaciones culturales de otros artistas con proyectos afines, generalmente, basadas en relaciones interpersonales sustentadas en amistad, compañerismo, confianza o afecto mutuo. Estas relaciones se vinculan al ámbito contextual donde están insertos. Por lo tanto, estas prácticas se asocian a la institucionalización de los grupos que se presentan dentro del circuito artístico. Enedina Ortega Gutiérrez (2012) revela que los creadores experimentan diversas formas, estilos de vida e innovaciones que llevan al desarrollo de organizaciones bajo un modelo de red. La etnografía observa que la red funciona como modelo de agregación, de asociación y contagio creativo estético, alrededor de proyectos de aprendizaje (de carácter creativo y/o empresarial) y multidisciplinarios (creativos y/o artísticos funcionales). Sin embargo, la base de estas redes sigue siendo la sociabilidad entre pares y sus espacios físicos y virtuales que los retroalimenta creativamente.

Finalmente, este apartado incluye las formas de trabajo de los artistas visuales. Es decir, enfocados, únicamente, en su práctica o si han tenido que incursionar de forma paralela en la curaduría, en la investigación, en la museografía o en algún otro rol. Ruth revela que la esencia del arte recae en la labor orgánica del artista, con su obra, así como con el compromiso que implica esta práctica al tener que trabajar en paralelo a la producción de la obra. Ella indica: "igual que todos los estudiantes, trato con mi obra propia, hago difusión cultural, ahí está el proceso y la ejecución del arte. Hay que hacer de todo y tienes que aprender a trabajar en paralelo" (Ruth, comunicación personal, 2016). Isabelle Graw (2014) explica que los perfiles profesionales ampliados reflejan, también, el "imperativo de conectividad" que obliga a los miembros del mundo del arte a colaborar continuamente. Al operar desde distintos roles, uno se ve obligado a esforzarse en todas direcciones, para no dejar pasar, digamos, ni la menor oportunidad de colaboración. Enrique categóricamente enuncia al artista contemporáneo como un profesional multitareas, que debe exigirse cubrir todos los rubros de su práctica, para insertarse de forma más eficiente en el mundo del arte.

Desde los sesenta ya se concebía al artista como multitareas, eso es algo básico para mí, eso tiene que ser. El artista tiene que ver por sí mismo, pensar en su trabajo, hablar con gente, tener relaciones públicas, administrarse, trabajar en otras cosas mientras le va llegando su lugar en el mundo del arte. Es parte del perfil del artista de mitad del siglo veinte para acá (Enrique, comunicación personal, 2016). 
Una forma de trabajo es el dividir el esfuerzo y los recursos en función de una producción artística, pero, la realidad es que no es así. Los artistas visuales tienen que saber jugar distintos papeles para lograr posicionar su obra, incluso, trabajar de manera paralela en otros proyectos, para gestionar mayores recursos. Tienen que convertirse en curadores, museógrafos, administradores, relacionistas públicos, entre otros. El artista que mejor sepa manejar estos rubros, además, de sus redes sociales digitales; aprovechar sus contactos en los circuitos artísticos y desarrollar procesos de autogestión podrá contar con herramientas sólidas para generar vínculos. Aunque algunos, también asumieron esto como una ventaja y no como un camino adicional por recorrer. Es decir, el poder conocer el lenguaje y la logística que gira en torno a la producción del arte permite una mayor eficacia profesional.

Una de las aproximaciones a la escuela de artes es su función como puente, ya que formaliza la creación de vínculos. El capital social no solo implica la red de relaciones sino la capacidad de trasladar los vínculos y movilizarlos, para generar beneficios específicos. No obstante, el principio de reconocimiento en un contexto homogéneo es la característica necesaria para que exista una transformación entre relaciones de cualquier tipo a relaciones de capital social. Esta transición está circunscrita a los recursos enlistados por cada artista y cómo los dirigen a través de los diferentes caminos, descritos en este apartado, para generar vínculos. Las distintas formas, señaladas por los entrevistados, para crear y movilizar los vínculos nacen en la pertenencia y los recursos que obtuvieron al ser egresados de FAV, lo cual les permite enlazar sus proyectos como artistas con la escena profesional.

\section{Aspecto 4. Intercambios}

El último aspecto en la construcción del capital social son los intercambios. El contenido de estos está orientado a intercambiar apoyo social, los cuales pueden ser materiales o simbólicos (Bourdieu, 1980). Los primeros se refieren a la ayuda material: monetaria, financiera o de información mientras el segundo rubro apunta a la dimensión emocional y afectiva. En ambos grupos se observa el proceso de consolidación del capital social, ya que representan una vinculación superior a lo contingente. Al llegar a este apartado, la clave es dedicar distintos recursos e invertir tiempo en movilizar el capital social para que pueda ser de largo plazo.

Los entrevistados mencionaron distintos tipos de intercambios, los cuales se agruparon como subcategorías dentro de los dos rubros que menciona Bourdieu. Dentro del primero, se encontraron cuatro distintas modalidades que, para fines de este proyecto, fueron denominados de la siguiente forma: intangibles, de red, oportunidades laborales y, finalmente, los intercambios materiales institucionales. En primer lugar, se encuentran los intangibles. Entre ellos se presenta el intercambio de tiempo, conocimiento, experiencia, información de becas y apoyos, información de trabajo, información acerca de convocatorias, información en 
redes sociales e invitación a montajes. Sobre este, Elma comenta: "la iniciativa privada con gente joven, que va empezando apoya mucho, porque saben lo difícil que es. Entre todos hacemos comunidad y apoquinamos algo, yo lo que mas pongo es tiempo, soy como una pequeña agencia que vincula gente (comunicación personal 2016).

La segunda clase se calificó como intercambios materiales de red, los cuales se refieren a los contactos de trabajo que se lograron vincular. En ellos se encontraron dos caminos básicos para conseguirlos. Uno era por medio de la FAV y del trabajo que los artistas realicen y el otro se daba a partir de eventos culturales, exposiciones o fiestas. La tercera tipificación, dentro de los intercambios materiales, es el rubro denominado como oportunidades laborales, que se materializa individual o colectivamente. En las primeras de estas oportunidades laborales se encuentran: el trabajo como docente, conferencista o exposición; trabajo en arteterapia; así como en eventos, cursos, talleres de logística y autogestión; clases de fotografía y de producción de moda; becas en CONARTE o en el Fondo Nacional para la Cultura y las Artes (FONCA). El segundo rubro se refiere a las oportunidades laborales de forma grupal, entre las más destacadas fueron, el trabajo colaborativo en festivales y exposiciones colectivas en espacios culturales. Néstor García Canclini (2012), en De la cultura postindustrial a las estrategias de los jóvenes, establece que:

los artistas declaran estar acostumbrados a organizarse en proyectos de corta y media duración. Algunos realizan emprendimientos independientes por convicción, pero la mayoría lo hace por necesidad. La creatividad y la innovación son dos rasgos altamente valorados para conseguir trabajo, lo cual contribuye a dar a sus actividades esta periodización frágil. La presión de lo instantáneo, lo que se descubre o se informa hoy, refuerza esta relación con la temporalidad veloz de las biografías: todo es efímero, renovable y luego obsoleto. Incluso los agrupamientos que organizan los jóvenes para poder trabajar. Las narrativas personales expresan esta visión inestable y de corto plazo. Además, los emprendimientos se despliegan como complementación de aprendizajes múltiples, relaciones de amistad junto a colaboraciones con grandes instituciones, competencia, autoempleo e inserción en redes preexistentes.

También hay que valorar, junto con la creatividad, las consecuencias de esta reorganización social y económica sobre la producción cultural. Si bien este modo de trabajar por proyectos, contratando y descontratando gente, permite a algunos liberarse de la rutina e innovar en tareas diversas y dinamiza la economía, también, perjudica a muchos. La extrema flexibilidad e inestabilidad laboral puede hacernos menos creativos y no saber cómo encarar las dificultades. La atención prestada a los comportamientos de los jóvenes permite registrar no solo su vulnerabilidad por el estrechamiento del mercado laboral, sino también su capacidad de agenciamiento 
La construcción del capital social en los egresados de

Artículos

la Facultad de Artes Visuales (FAV) en Monterrey, México

y el uso innovador de capitales culturales y sociales. No están apresados en la crisis de la industria. Se mueven hacia horizontes no previstos por el desarrollo clásico de la misma. Se esboza así un mapa más abierto e incluyente (García, 2012, pp.14 -22).

Los artistas visuales buscan formas flexibles de agrupación en las que los procesos colaborativos sean compatibles con la individualidad y los proyectos personales de sus integrantes. Esto permite participar, simultáneamente, de un número amplio de iniciativas artísticas que, por lo general, no resultan conformaciones estables en el tiempo, sino, más bien, proyectos efímeros que valoran el movimiento de la escena y la multiplicidad de sus propuestas (Gerber \& Pinochet, 2012, p. 60).

La cuarta categoría son los intercambios materiales institucionales, que se refiere a las cuestiones propias de una obra, su ejecución, exposición y difusión. Entre los intercambios comentados estuvieron: los materiales para producir, la gestión de obra y espacios, curaduría, la estrategia de mercado, préstamo de espacio, proveedores, espacios de circulación. Paralelamente a estos intercambios materiales, suceden los simbólicos, los cuales, se observó, que consolidan los intercambios previamente explicados. Estas representaciones fueron utilizadas para enfatizar el valor y la carga emotiva de alguna relación institucional o entre artistas. Entre los más destacados se encontraron, el amor, la amistad, el trabajo en equipo, la empatía, la generosidad, los sueños compartidos, la confianza, la honestidad, el apoyo y la comprensión. El capital social tiene que ver con un aspecto afectivo, por lo tanto, la proyección de los artistas en los distintos espacios está ligada a este aspecto. El bienestar social, emocional y afectivo está vinculado a sus grupos de apoyo y trabajo, lo cual determinará cómo y cuáles vínculos fortalecerán. Este tipo de relaciones son los hilos invisibles sostenidos por la adaptación, afinidad y el aval de la institución, en este caso de la FAV. Adán comenta "la FAV sí te legitima mucho, cuando eres egresado pero mucho más cuando eres alumno, porque tienes los contactos de primera mano de los maestros y compañeros" (comunicación personal, 2017)

Finalmente, los valores que son compartidos fueron los que estaban centrados en crear redes de contacto y formación de equipo, tales como objetivos en común, persistencia, servir como puente, generar comunidad, valores comunitarios, disciplina, responsabilidad y consistencia. De acuerdo con lo que manifestaron los entrevistados, el contenido cooperativo y recíproco de las relaciones en el campo del arte es alto, debido a los equipos de trabajo y el intercambio mutuo entre instituciones e individuos. Estas redes de relaciones entre individuos y grupos asumen el reconocimiento del otro, y transforman los recursos en vínculos e intercambios de larga duración. De tal forma, las estrategias de reproducción social tienen que ver con la trayectoria del capital social del individuo y del grupo o los grupos con los cuales se conecte. El capital social de los artistas visuales se moviliza y se 
traslada de acuerdo a la propuesta y discurso que cada uno maneje, lo cual permite que el panorama de trabajo involucre proyectos de producción artística o creativos con un amplio espectro de posibilidades.

\section{Conclusiones}

El capital social es un recurso del agente a partir de los recursos que sus relaciones poseen. Este concepto integra las dinámicas relacionales en la explicación de los fenómenos sociales. Bourdieu lo perfila como un medio para conocer la trayectoria de un agente social. Por esta razón, se empleó para explorar el proceso de su construcción en el campo del arte, con el objetivo de indagar en la proyección profesional de los artistas visuales egresados de FAV. Bajo este contexto, se vislumbra a la Facultad de Artes como un espacio mediador y, sobre todo, como la plataforma que define la construcción del capital social de los artistas visuales.

Debido a los cambios que el arte ha registrado desde la mitad del siglo XX, se han articulado nuevas salidas profesionales para los artistas visuales y se ha reconfigurado la suposición que implica artista-galería-coleccionista-museo. Por lo tanto, en la institución del arte se han tenido que contemplar circuitos artísticos poco habituales, como los mercados de diseño, barrios públicos, festivales, entre otras instancias laborales, donde los artistas han encontrado un espacio. La capacidad de relacionarse y la forma en la que se construyen redes de contacto ha sido remarcada como una de las estrategias principales para lograr una inserción profesional satisfactoria. Por esta razón, se considera valioso conocer el proceso de construcción del capital social. Aunque no se puede asegurar como una fórmula infalible, sí ofrece las bases para establecer la generación y movilización eficaz de los recursos disponibles como egresado de una escuela de artes.

El proceso de construcción del capital social está sujeto a distintas condiciones epocales, ya que el caso de cada uno de los entrevistados es distinto. No obstante, estas generalidades ilustran cómo se han desarrollado los factores más relevantes y los retos implicados. Uno de los hallazgos más importantes fue descubrir el valor del trabajo en equipo. Compartir objetivos en común, a través de proyectos colectivos fue una de las herramientas con mayor mención durante el proceso de construcción del capital social. Los artistas visuales y los agentes del círculo del arte son los que se encargan de una auténtica creación continua de relaciones. Por lo tanto, gozar de la confianza es un intercambio simbólico que define las relaciones que se pueden movilizar eficazmente. Estos intercambios son indispensables para obtener el mejor rendimiento del capital, por ello, son los que determinan los desplazamientos en el espacio social, es decir, las trayectorias de los artistas visuales. Sin embargo, la acumulación de este capital no posee una incorporación automática a la proyección profesional, es su movilización lo que lo logra. Uno de los aspectos empíricamente 
La construcción del capital social en los egresados de

Artículos

la Facultad de Artes Visuales (FAV) en Monterrey, México

observados es que la proyección profesional es distinta para cada artista. Pero, la mayoría enuncia el éxito laboral en términos que implican no solo la subsistencia económica, sino la afinidad afectiva, por lo cual, sus redes de contacto están sujetas a estas condiciones.

El arte como fenómeno social está relacionado a los circuitos de mayor visibilidad e impacto. La dimensión local coexiste con esta referencia a procesos globales al buscar una identificación regional. Monterrey, y muchas ciudades del mundo, han entrado en la tendencia de las industrias creativas, donde el diseño, la publicidad, el arte y la comunicación son un sector en constante crecimiento y comunicación. Las oportunidades de emprendimiento y laborales se han multiplicado en esa área. Hay una mayor demanda por estas disciplinas, debido a las necesidades culturales y estéticas que se han ampliado, así como a los estrechos horizontes que existen, actualmente, en la aplicación de las prácticas artísticas, diseño, ilustración o animación. Eduardo Ramírez (2011) menciona en el ensayo Monterrey, capital e imagen, que dentro de los procesos de reconversión de la industria neolonesa, para integrarse al circuito económico global al final del milenio, Monterrey siguió la tendencia de las Ciudades del Conocimiento. Este modelo implica pasar de la producción material a invertir en educación, cultura, arquitectura, diseño, medios de comunicación y socialización.

De estas implicaciones nace la importancia de estudiar a una de las escuelas que forma a estos profesionistas, ya que su desarrollo ha repercutido en la agenda cultural y la escena profesional del arte, al interactuar con los sectores industriales y comerciales de la localidad. Si bien, el nacimiento y desarrollo de la Facultad de Artes Visuales va desde hace un par de décadas a la actualidad es necesario resaltar los aciertos que ha tenido. Entre ellos, el ser la pionera en tomar el reto de formar especialistas en artes dentro de una ciudad como Monterrey, donde la lógica empresarial y de negocios pudo desplazar a esta práctica a un pasatiempo, un saber técnico o una terapia. Por otro lado, se encuentra el compromiso que ha inculcado en sus egresados de sortear esos obstáculos a nivel profesional. Así mismo, el plantear su producción en términos de congruencia con su tiempo, sus alcances y su contexto.

Resulta clave la comprensión de la FAV como un enlace entre el mundo académico y el profesional, también, como una plataforma que favorece la construcción de una propuesta artística y de un discurso claro. Además, todos admitieron explícita, o implícitamente, el valor que tiene en su currículum y en su formación individual como artistas. Bajo esta perspectiva, se reconoce el valor que ha tenido la FAV al continuar con esta licenciatura de arte, pese a tener generaciones que, en ocasiones, no superan los cinco alumnos. Es un esfuerzo que sostienen como muestra del compromiso con la formación integral en el arte, la cual mantienen con la localidad y con el país, como universidad pública. Formar artistas visuales con el imaginario propio de un regiomontano, que convive con la vibrante cultura mexicana y las globalizadas tendencias estadounidenses es un contexto que no se puede desaprovechar para conformar potentes narrativas artísticas.

ESCENA. Revista de las artes, 2019, Vol. 79, Núm. 1 (julio-diciembre), pp. 119-144 
La naturaleza del arte es distinta, así como su ejercicio y sus criterios de enseñanza e investigación. No obstante, se ha convertido en una disciplina con mayores aplicaciones y aportes a la vida diaria. El objetivo de una escuela de artes, no solo gira en torno a la dotación de imaginarios, comprensión de técnicas o confrontación con la historia del arte. Sino, también estimula la producción y facilita las herramientas para insertarse en su particular contexto. Con esto, se confirma la importancia de las licenciaturas en artes. No es un oficio ni un saber técnico. El espacio profesional que ha logrado tiene que ver con las demandas culturales de una sociedad. La herencia que corresponde al arte local evidencia una serie de necesidades por cubrir. Aunque, también, señala un espacio de riqueza creativa, propuestas artísticas y proyectos por desarrollar.

\section{Referencias}

Becker, H. (2009). Los mundos del arte. Sociología del trabajo artístico (Trad. Joaquín Ibarburu). Bernal, Argentina: Universidad Nacional de Quilmes.

Bourdieu, P. (1972). Esquisse d'une theorie de la pratique. Genève, Paris: Editions Droz.

Bourdieu, P. (1980). El capital social. Notas provisorias. Actes de la recherche en sciences sociales, 31, 224.

Bourdieu, P. (1986). Las formas del capital. Handbook of Theory and Research for the Sociology of Education. Nueva York: Greenwood.

Corona, I. (2011). La avanzada regia: Monterrey's alternative music scene and the aesthetics of transnationalism. En Alejandro L. Madrid (Ed.), Transnational encounters, Music and performance at the U.S. - Mexico border (252-284). New York, Oxford University Pres.

Dickie, G. (1997). El círculo del arte. Una teoría del arte. Barcelona: Paidós.

García, N. (2012). De la cultura postindustrial a las estrategias de los jóvenes. En García, N. \& Cruces, F. \& Urteaga, M. (Eds.), Jóvenes, Culturas urbanas y redes digitales (3-24). Barcelona: Editorial Ariel, S.A. .

García, N. \& Piedras, E. (2013) Jóvenes creativos, estrategias y redes culturales. México: Universidad Autónoma Metropolitana.

Gerber, V. \& Pinochet, C. (2012). Compendio para ciegos (o dónde buscar el relato de una generación invisible). En García, N. \& Urteaga, M. (Eds.), Cultura y desarrollo: una visión crítica desde los jóvenes (37-90). Madrid: Fundación Carolina.

Graw, I. (2014). ¿Cuánto vale el arte? Mercado, especulación y cultura de la celebridad. (Trad. 
La construcción del capital social en los egresados de Artículos la Facultad de Artes Visuales (FAV) en Monterrey, México

de Cecilia Pavón y Claudio Iglesias). Argentina: Ed. Mardulce.

Heinich, N. (1998). Le triple jeu de l'art contemporain. Paris: Minuit.

Herrera, A. (2004). La condición de la producción del arte contemporáneo en Monterrey. En Herrera, A. Sierra, B. Ruiz, E. (Ed.), Transferencias, convenciones y simulacros. Diálogos para una visión y una interpretación de las artes visuales de Monterrey (pp. 08-19). Monterrey: Universidad Autónoma de Nuevo León.

Liot, F. (2004). Le métier d'artiste: les transformations de la profession artistique face aux politiques de soutien à la création. Francia: Editions L'Harmattan.

Moureau, N. \& Zenou, B. (2014). El capital social, el arte contemporáneo y las carreras. REDESRevista hispana para el análisis de redes sociales, 25(2), 106 - 124.

Ortega, E. (2012). Aprendices, emprendedores y empresarios. En García, N. \& Cruces, F. \& Urteaga, M. (Eds.), Jóvenes, Culturas urbanas y redes digitales (109-140). Barcelona: Editorial Ariel, S.A.

Ramírez, E. (2011). Monterrey, capital e imagen. En Alonso, L., Cabrera, R., Ramírez, E. \& Juárez, L. (Editores). Volverse y mirar a Monterrey: apuntes desde el posgrado de artes visuales de la Universidad Autónoma de Nuevo León. Monterrey: Universidad Autónoma de Nuevo León.

Ramírez, J. (2005). Tres visiones sobre capital social: Bourdieu, Coleman y Putnam. Acta Republicana. Política y Sociedad, 4, 21-36.

Rubiano, E. (2013, 24 de abril). Autonomía y sujeción: el arte y los artistas en el contexto de la gestión creativa. [Ponencia]. En Congreso Producir la Escena: Modus Operandi \#9. Colombia: Universidad de los Andes.

Salinas, C. (2015). Paisaje Industrial de Monterrey: el discurso sobre la nueva imagen de la ciudad [Ponencia presentada en Financiarte, Conarte]. Centro Eugenio Garza Sada, Monterrey, México.

Sierra, B. (2004). 3. En Herrera, A., Sierra, B. \& Ruiz, E. (Eds.). Transferencias, convenciones y simulacros. Diálogos para una visión y una interpretación de las artes visuales de Monterrey (20-53). Monterrey: Universidad Autónoma de Nuevo León.

Vivar, C. \& Arantzamendi, M., Lopez-Dicastillo, O. \& Gordo, L. (2010). La Teoría Fundamentada como Metodología de Investigación Cualitativa en Enfermería Index Enferm, 4(19), 283-288.

Zenou, B. (2009). Le Capital Social comme un Potentiel d'Interaction Coopérative: le cas des Relations Intergénérationnelles Familiales. [Tesis de doctorado]. Universidad de Montpelier, Montpellier, Francia.

ESCENA. Revista de las artes, 2019, Vol. 79, Núm. 1 (julio-diciembre), pp. 119-144 